\title{
The timbre of compound tones consisting of four amplitude modulated waves of different frequency regions
}

\author{
Shin-ichiro Iwamiya* and Manabu Okamoto** \\ *Department of Acoustic Design, Kyushu Institute of Design, \\ 4-9-1, Shiobaru, Minami-ku, Fukuoka, 815 Japan \\ **NTT Human Interface Laboratories, \\ 1-2356, Take, Yokosuka, 238-03 Japan
}

(Received 27 June 1995)

\begin{abstract}
The timbre of compound tones consisting of four amplitude modulated waves is examined by psychoacoustical experiments using a similarity judgement of timbre. The obtained data are analyzed by a multi-dimensional scaling (MDS) method. The MDS solutions are different according to the frequency regions of the AM waves. When the frequency region is low and there is one AM wave within the bandwidth of the auditory filter, envelope correlations between AM waves are a determinant factor of timbre. However, the three dimensional MDS solution suggests that all the envelopes do not affect the timbre in the same manner. The effect of the lowest AM wave is not clear. As the frequency region is higher, the effect of the envelope correlation is less dominant. The effect of the depth of amplitude modulation of the compound waves then becomes dominant. When there are two AM waves within the bandwidth of the auditory filter, we no longer see the effect of the envelope correlation between AM waves. In this case, the correspondence of the shape of the compound envelopes of two AM waves affect the timbre.
\end{abstract}

Keywords : Timbre, Multi-dimensional scaling (MDS) method, Amplitude envelope correlation, Depth of amplitude modulation, Auditory filter

PACS number: 43. 66. Jh

\section{INTRODUCTION}

"Amplitude envelope" is one of the physical determinant factors of timbre. In particular, combinations of amplitude envelopes of each harmonic contribute to the timbre of violin and vocal vibrato sounds (Fletcher and Sanders, 1967; Sundberg, 1982).

In the previous study, the timbre of compound tones consisting of three amplitude modulated (AM) waves was examined by psychoacoustical experiments to clarify the effect of combinations of amplitude envelopes (Iwamiya, 1995). The results showed that the envelope correlations between adjacent AM waves were the determinant factor of tim- bre. The effects of the correlation between the first and the second AM waves and those between the second and the third AM waves were observed. These effects on timbre are considered to be based on the function of the auditory system to detect the amplitude envelope correlation between the output signals of the different auditory filters. There seem to be "envelope correlators" after the auditory filters. Several studies show the evidences of existence of "amplitude envelope correlator" (Richards, 1987, 1988; Yost and Sheft, 1989; Green et al., 1990 ; Moore and Emmerich, 1990).

In the previous study (Iwamiya, 1995), we obtained important knowledge about the relationship between amplitude envelopes and timbre. 
However, these results were based on the experiment using the sound stimuli consisting of three AM waves with the carrier frequencies of 440,880 , and $1,320 \mathrm{~Hz}$.

If there are more than three AM waves, "do all the envelope correlations between adjacent AM waves affect the timbre?" In real musical sounds, there are many components. It may be difficult for the auditory system to use all the envelope correlations between the components.

Also, if the frequency region of AM waves is different, "can we obtain a similar tendency?" The envelope correlations are determined from the output signals of the auditory filters. The bandwidth of the auditory filter depends on the frequency of the acoustic signal (Zwicker et al., 1957). Then the experimental results may be affected by the frequency region of the AM waves.

To clarify these issues, in this study, the effect of amplitude envelopes of AM waves on the timbre of the compound tones consisting of four AM waves is examined as a function of the frequency region of AM waves.

\section{EXPERIMENTAL METHOD}

The sound stimuli of the present experiment are defined as ;

$$
\sum_{i=L}^{L+3}\left[1+\cos \left(2 \pi f_{\bmod } t+\theta_{i}\right)\right] \cos \left(2 \pi i f_{\mathrm{c}} t\right) .
$$

The carrier waves of each AM wave had the same amplitude. The fundamental frequency $\left(f_{\mathrm{c}}\right)$ of the carrier waves was $440 \mathrm{~Hz}$. The modulation wave was sinusoidal and the modulation frequency $\left(f_{\text {mod }}\right)$ was $8 \mathrm{~Hz}$. The modulation depth ([maximum amplitude - minimum amplitude $] /[$ maximum amplitude+minimum amplitude]) of each AM wave was $100 \%$. The duration of the sound stimuli was $1.6 \mathrm{~s}$. They included $100-\mathrm{ms}$ rise/fall time shaped with a linear function.

Four experiments were conducted according to the values of $L$; they were $L=1, L=5, L=9$, and $L=13$. The carrier frequencies of the $A M$ waves were $440-1,760 \mathrm{~Hz}$ at $L=1,2,200-3,520 \mathrm{~Hz}$ at $L=$ $5,3,960-5,280 \mathrm{~Hz}$ at $L=9$, and $5,720-7,040 \mathrm{~Hz}$ at $L=13$. The value of $L$ defines the frequency region of AM waves.

The initial phase of amplitude modulation was 0 degrees for the lowest $\mathrm{AM}$ wave $\left(\theta_{\mathrm{L}}\right)$, and 0 or 180 degrees for the other AM waves. The phase of modulation and carrier waves was determined at the stage of the electrical signal.

The sound stimuli are defined by four-digit numbers for the sake of convenience. Each number shows the initial phase of the modulation waves. The highest digit shows $\theta_{\mathrm{L}}$ and the lowest digit shows $\theta_{\mathrm{L}+3}$. Similar to the previous study, "0" means 0 degrees and " 2 " means 180 degrees. For example, "0002" represents that $\theta_{\mathrm{L}}=\theta_{\mathrm{L}+1}=\theta_{\mathrm{L}+2}=0$ degrees and $\theta_{\mathrm{L}+3}=180$ degrees. There are eight combinations of the initial phase of the modulation waves.

The sound stimuli were generated on a computer (TEAC PS-9000 300M) and played through a digital-to-analog converter (TEAC PS-9353). They were presented diotically via headphones (Stax SR-X/MK3) as pairs at a level of $70 \mathrm{~dB}$ SPL. The interstimulus interval between each stimulus pair was $200 \mathrm{~ms}$.

The task of the subjects was to judge similarity of timbre on a 7-step scale between the pairs. The subject's response was obtained by the keyboards of a portable computer (Sharp PC-1600K) which was connected with the main computer (TEAC PS- 9000 $300 \mathrm{M})$. There was no time limit of judgment. After the response was obtained, the next pair was presented. All the combinations of the eight sound stimuli were presented randomly.

In order to examine the effect of pitch and loudness difference on timbre perception, the authors compared pitch and loudness of all these stimulus pairs. As a result, no pitch and loudness difference among the stimuli was observed for each $L$-value

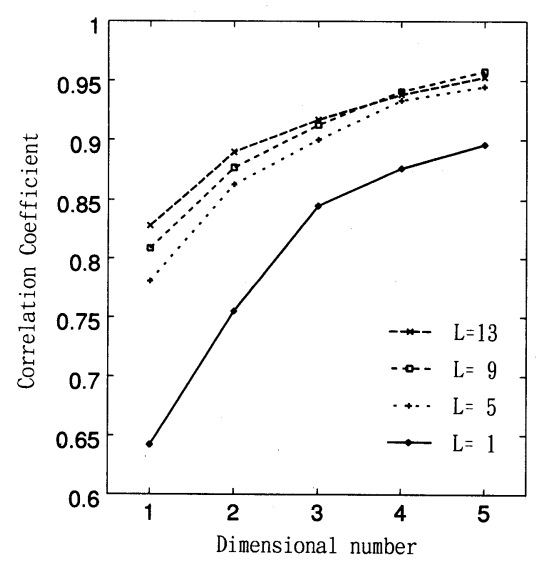

Fig. 1 Correlation coefficients of INDSCAL solutions as a function of the dimensional number for each value of $L$. 
condition.

Six subjects with normal hearing, five males and one female, aged 21 to 26 years old, participated.

\section{RESULTS AND DISCUSSION}

The obtained similarity matrix of the sound
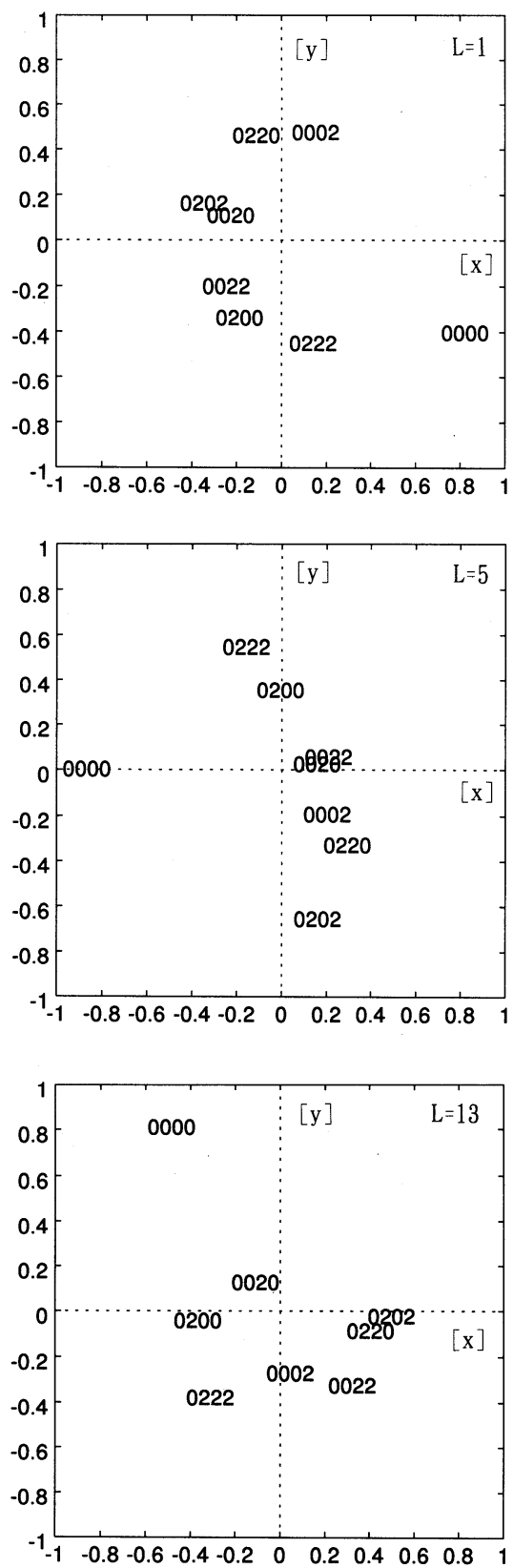

stimuli was analyzed by a multi-dimensional scaling method, called INDSCAL (Arabie et al., 1987).

The correlation coefficient between the scalar products derived from the original similarity matrix and those derived from the INDSCAL solution is taken as the criteria of INDSCAL solution (Arabie
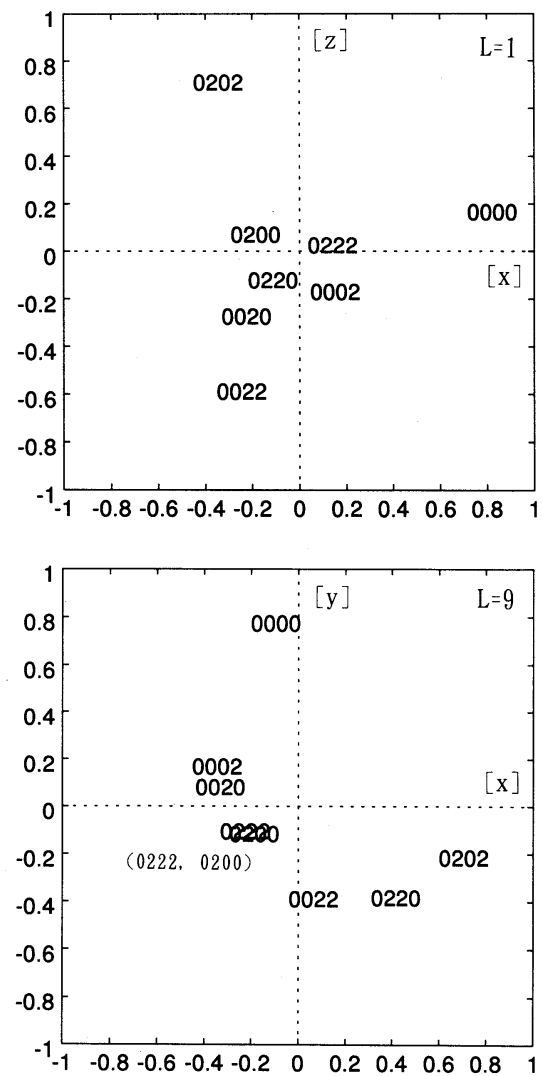

Fig. 2 Three dimensional INDSCAL solution at $L=1$, and two dimensional INDSCAL solutions at $L=5$, $L=9$, and $L=13$. 
et al., 1987) for determination of the number of dimension. Figure 1 shows the correlation coefficients as a function of the dimensional number for each value of $L$. Because of the saturation of increase of the correlations, the three dimensional solution was chosen at $L=1$, and the two dimen-
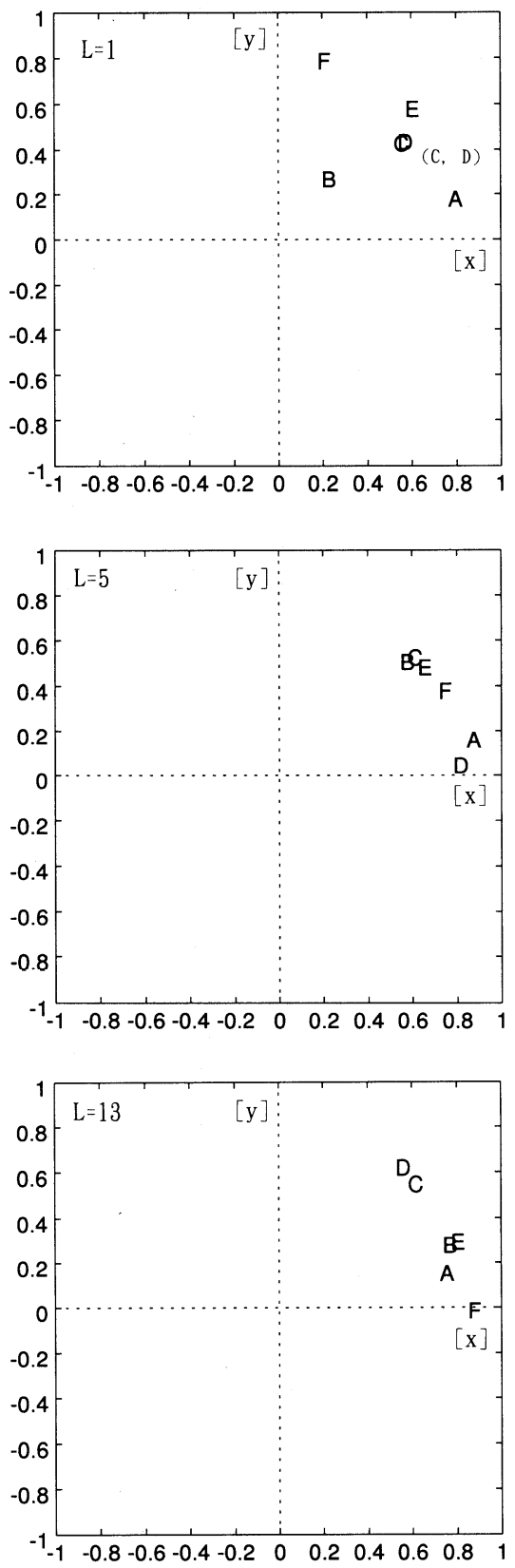

Fig. 3 Individual weights of the subjects for each dimension at $L=1, L=5, L=9$, and $L=13$. Each letter shows a subject. sional solutions were chosen in the other conditions. The correlation coefficient of each solution is 0.85 at $L=1,0.86$ at $L=5,0.88$ at $L=9$, and 0.89 at $L=13$.

Figure 2 shows the configuration of the sound stimuli for each condition. If coordinates of stimuli overlap, the figures are shown in the paren-
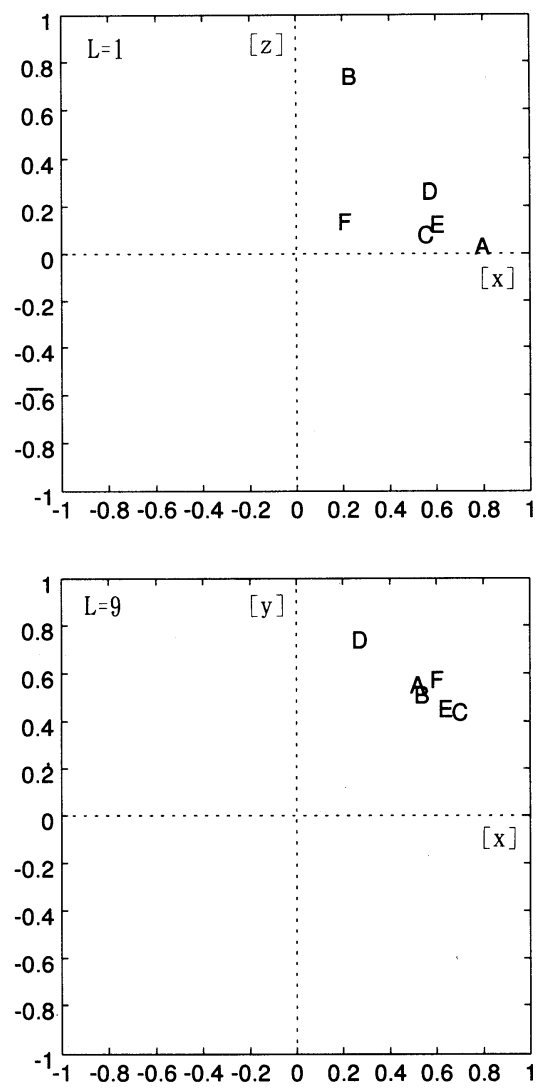
theses. Figure 3 shows the individual weights of the subjects for each condition.

\subsection{Configuration of the Sound Stimuli in Each Condition and the Determinant Physical Fac- tor of Timbre}

In the case of $L=1$, we can see the effects of the envelope correlations between adjacent AM waves. In Fig. 2, the numbers of the middle two digits of the sound stimuli are the same (" 00 " or " 22 ") when the $x$-coordinate is positive. They are different ("02" or "20") when it is negative except "0220." Among the sound stimuli in the negative region of the $x$-axis, "0220" is located at the most positive side.

As a result, the sound stimuli are divided into two groups along the $x$-axis according to the numbers of the middle two digits: They are $(0202,0020,0022$, and 0200$)$ and $(0220,0002,0222$, and 0000$)$. The numbers are different in the former group and the same in the latter group. The second amplitude modulation and the third amplitude modulation are out of phase in the former group and in phase in the latter group. Therefore, the location of the sound stimuli along the $x$-axis is related to the phase difference between the second and the third amplitude modulations.

The sound stimuli are also divided into two groups along the $y$-axis : They are $(0220,0002,0202$, and 0020$)$ and $(0022,0200,0000$, and 0222$)$. The third amplitude modulation and the fourth amplitude modulation are out of phase in the former group and in phase in the latter group. Therefore, the location of the sound stimuli along the $y$-axis is related to the phase difference between the third and the fourth amplitude modulations.

These results show the effects of envelope correlations among the three highest AM waves on timbre. When amplitude modulations are in phase, the correlation coefficient between the amplitude envelopes is +1 . When amplitude modulations are out of phase, it is -1 . As shown in the previous study (Iwamiya, 1995), envelope correlations are the determinant factor of the sound stimuli configuration in the MDS solution. However, the effect of the lowest AM wave is not shown on the $x-y$ plane.

In Fig. 3 (at $L=1$ ), subjects $\mathrm{C}, \mathrm{D}$, and $\mathrm{E}$ show similar weight for the $x$ and $y$-axis. Subject $\mathrm{A}$ shows small weight for the $y$-axis and subject $\mathrm{F}$ shows small weight for the $x$-axis. Subject B shows small weight for both the axes. There are individ- ual differences in which envelope correlation is more effective on timbre perception.

There is a rough tendency that when the first and the second amplitude modulations are in phase, the $z$-coordinates of the sound stimuli are more negative than when they are out of phase. However, this effect is not consistent. Although the first and the second amplitude modulations are in phase, the $z$-coordinate of the sound stimulus " 0000 " is more positive than those of "0200," "0222," and " 0220 " whose first and second amplitude modulations are out of phase. The effect of the lowest AM wave is not clear.

Furthermore, only subject B shows large weight for the $z$-axis. The other subjects show small weight for it. The third dimension $(z)$ does not seem to be meaningful for the majority of the subjects. On the other hand, subject B shows small weight for the $x$ and $y$-axis.

Consequently, the three dimensional solution suggests that all the envelopes do not affect the timbre in the same manner.

In the case of $L=5$, we can also see the effect of the phase relation between the third and the fourth amplitude modulations. When the third and the fourth amplitude modulations are in phase, the $y$-coordinate of the sound stimuli is positive. When they are out of phase, the $y$-coordinate is negative except "0020." There is one exception, however, the sound stimuli are divided into two groups according to the envelope correlation between the third and the fourth AM waves along the $y$-coordinate.

In this condition, however, the effect of the envelope correlation between the other AM waves cannot be observed. We can see the effect of the amplitude modulation depth of the whole sound along the $x$-axis, rather than that of envelope correlation between the other AM waves.

The sound stimulus " 0000 " is clearly separated from the others. " 0000 " is the only stimulus whose depth of amplitude modulation is $100 \%$. In general, the stimuli with $0 \%$ modulation depth $(0022$, 0220 , and 0202) are located at the farthest position from " 0000 " along the $x$-axis. The stimuli with $50 \%$ modulation depth $(0222,0200,0020$, and 0002) tend to be in the middle between the stimuli with $0 \%$ modulation depth and the stimulus with $100 \%$. However, the configuration differences between the stimuli with $0 \%$ and $50 \%$ depth are not clear in this 
condition.

The subjects show similar weight for the $x$-axis, and there are individual differences in the weight for the $y$-axis (Fig. 3). For all the subjects the depth of amplitude modulation affects timbre perception. For subjects $\mathrm{A}$ and $\mathrm{D}$, the envelope correlation between the third and the fourth AM waves is not effective.

We can also see the effect of amplitude modulation depth along the $x$-axis in the case of $L=1$. In general, the larger the modulation depth, the more positive the $x$-coordinate. However, similar to the condition of $L=5$, the configuration differences between the stimuli with $0 \%$ and $50 \%$ depth are not clear. The sound stimulus " 0220 " is more positive than " 0020 " and "0200," although the modulation depth of " 0220 " is smaller than those of " 0020 " and "0200." On the other hand, the division of the stimuli based on the phase difference between the second and the third amplitude modulations shows no exception. Then, at $L=1$, the envelope correlation between the second and the third AM waves, is considered to be the more dominant determinant factor of the $x$-coordinates.

In the case of $L=9$, we no longer see the effect of the envelope correlation between AM waves. The effect of modulation depth of the whole sound is clearly observed. The stimuli are divided into three groups according to the modulation depth of $100 \%$, $50 \%$, and $0 \%$ : They are $(0000),(0002,0020,0222$, and 0200), and (0022, 0220, and 0202). The $y$ coordinates nearly correspond with this grouping.

The subjects show similar weight for the $x$ and $y$-axis except subject D (Fig. 3). Subject D shows small weight for the $x$-axis.

This similar tendency is obtained in the case of $L=13$. We still cannot see the effect of the envelope correlations between AM waves in this case. The stimuli are also divided into three groups according to the modulation depth of the whole sound: They are (0000), (0002, 0020, 0222, and $0200)$, and $(0022,0220$, and 0202). This grouping corresponds to the diagonal line from the second quadrant to the fourth quadrant.

The subjects show similar weight for the $x$-axis, and there are individual differences in the weight for the $y$-axis (Fig. 3). In this condition, however, the meanings of each axis are not clear, as the results are interpreted by the diagonal line on the $x-y$ plane.
3.2 The Effect of the Frequency Region of the AM Waves

The effects of the envelope correlations between adjacent AM waves are observed in the cases of $L=$ 1 and $L=5$. However, they are not observed in the cases of $L=9$ and $L=13$.

The differences of the experimental results according to the values of $L$ are considered to be related to the number of the AM waves within the bandwidth of the auditory filter in each condition. Although the frequency difference between the carrier frequencies of adjacent AM waves is constant, the bandwidth of the auditory filter increases as the carrier frequency is higher. Then the number of AM waves within the bandwidth of the auditory filter is different according to the carrier frequencies of the AM waves. There is one AM wave within the bandwidth of the auditory filter at $L=1$, one or two AM waves at $L=5$, two AM waves at $L=9$, and two or three AM waves at $L=13$ (Zwicker et al., 1957).

When there is one AM wave within the bandwidth of the auditory filter, the envelope correlator of the auditory system can detect the envelopes of each AM wave independently. Then the envelope correlation between AM waves affects the timbre. When there are more than two AM waves within the bandwidth, the output of the auditory filter is the composition of the AM waves. Then the envelope correlator cannot make use of information on the envelopes of each AM wave respectively.

As the frequency region of the AM waves is higher ( $L$ is larger), the effect of the envelope correlations on timbre is smaller. Consequently, the effect of the modulation depth of total sounds becomes more dominant. Because " $L=5$ " is the critical condition, both the effects are shown.

Furthermore, there seems to be another key which affects the timbre of the compound tones consisting of four AM waves in the case of $L=9$. The sound stimuli are divided into two groups according to the $x$-coordinates: They are $(0002,0020,0200$, and $0222)$ and $(0000,0022,0202$, and 0220). In the former group the compound envelopes of the lower and the higher AM wave pairs have different shapes, sinusoidal and flat, or flat and sinusoidal. For example, for " 0020 ", the compound envelope of the lower AM wave pair ("00") is sinusoidal, and that of the higher AM wave pair ("20") is flat. On the latter group, the compound envelopes have the same 
shapes, sinusoidal and sinusoidal, or flat and flat. For example, "0022" is a combination of sinusoidal envelopes and " 0202 " is a combination of flat envelopes.

When there are two AM waves within the bandwidth of the auditory filter, the correspondence of the shapes of the compound envelopes of two AM waves affect the timbre. There seems to be an auditory process to detect the envelope shapes of outputs of the auditory filters and compare them.

When the envelope shapes of output signals of the auditory filters are the same (at $L=1$ ), the envelope correlation between the output signals of the auditory filters is a determinant factor of timbre. When the envelope shapes of outputs of the auditory filters are not the same (at $L=9$ ), their envelope correlation is not a determinant factor. In this case, correspondence of the envelope shapes becomes a determinant factor.

In the case of $L=13$ we can also see a similar tendency. The sound stimuli are divided into two groups by the diagonal line from the second quadrant to the fourth quadrant. The members of each group are the same as those in the case of $L=9$. However, because there are three AM waves in the auditory filters in the higher frequency region at $L=$ 13 , the grouping is not clear.

\section{CONCLUSION}

The effect of the amplitude envelopes of AM waves on the timbre of the compound tones consisting of four AM waves is examined as a function of the frequency region of the AM waves. A multidimensional scaling technique was applied to analysis of the timbre of these sounds.

When the frequency region is low and there is one AM wave within the bandwidth of the auditory filter, envelope correlations between the AM waves are a determinant factor of timbre. However, the three dimensional MDS solution suggests that all the envelopes do not affect the timbre in the same manner. The effect of the lowest AM is not clear.

As the frequency region is higher, the effect of the envelope correlations is less dominant. The effect of the depth of amplitude modulation of the compound waves then becomes dominant. When there are two AM waves, we no longer see the effect of the envelope correlations between the AM waves. In this case, the correspondence of the shape of the compound envelopes of two AM waves affect the timbre.

\section{REFERENCES}

Arabie, P., Carroll, J. D., and DeSarbo, W. S. (1987). Three-way Scaling and Clustering (Sage Publications, Inc., Newbury Park).

Fletcher, H. and Sanders, L. C. (1967). "Quality of violin vibrato tones," J. Acoust. Soc. Am. 41, 1534-1544.

Green, D. M., Richards, A. M., and Onsan, Z. A. (1990). "Sensitivity to envelope coherence," J. Acoust. Soc. Am. 87, 323-329.

Iwamiya, S. (1995). "The effect of amplitude envelopes of each amplitude modulated wave on the timbre of compound tones consisting of three amplitude modulated waves," J. Acoust. Soc. Jpn. (E) 16, 21-27.

Moore, B. C. J. and Emmerich, D. S. (1990). "Monaural envelope correlation perception, revised : Effects of bandwidth, frequency separation, duration, and relative level of the noise bands," J. Acoust. Soc. Am. 87, 2628-2633.

Richards, V. M. (1987). "Monaural envelope correlation perception,” J. Acoust. Soc. Am. 82, 1621-1630.

Richards, V. M. (1988). "Components of monaural envelope correlation perception," Hear. Res. 35, 47-58.

Sundberg, J. (1982). "Perception of singing," in The Psychology of Music, D. Deutch, Ed. (Academic Press, New York), Chap. 3, pp. 59-98.

Yost, W. A. and Sheft, S. (1989). "Across-critical-band processing of amplitude-modulated tones," J. Acoust. Soc. Am. 85, 848-857.

Zwicker, E., Flottorp, G., and Stevens, S. S. (1957). "Critical bandwidths in loudness summation," J. Acoust. Soc. Am. 29, 548-557. 